\title{
A RUST INTENSITY RECOGNITION APPROACH FOR STEEL BRIDGE SURFACE COATING IMAGES BASED ON ARTIFICIAL NEURAL NETWORKS
}

\author{
Po-Han Chen*, Heng-Kuang Shen, and Luh-Maan Chang \\ Department of Civil Engineering, National Taiwan University, Taipei, Taiwan \\ * Corresponding author (pohanchen@ntu.edu.tw)
}

\begin{abstract}
One of the traditional methods to assess the quality of the coating on the steel bridge surface is calculating the ratio of rust to the total area of coated surface. In North America, digital image processing has been applied on quality assessment of steel bridge coating since late 1990s due to the difficulty of rust area quantification by human vision. Most of the previously developed systems segment images into two groups, rust and background, before assess the rust ratio. These systems are usually lack of the ability to recognize the intensities of rust. In order to provide a flexible assessment, one pioneer method called adaptive ellipse approach (AEA) was proposed in 2009 to classify the images into three groups, background, rust, and mild-rust. However, even the three-group system is still weak at describing gradually changed colors and thus unable to classify those colors automatically. In this paper, we segmented the image into several clusters based on the similarity of colors, and used the root-mean-square standard deviation (RMSSTD) to measure the similarity of each cluster. Artificial Neural Networks was applied to choose the best number of clusters based on the values of RMSSTD. This paper models a rust intensity recognition approach to choose the most suitable number of clusters for describing rust intensities, trying to overcome a hurdle that two- or three-group systems could not resolve.
\end{abstract}

Keywords: Coating Defect Recognition, Color Image Processing, K-Means, Artificial Neural Network, Root-mean-square Standard Deviation (RMSSTD)

\section{INTRODUCTION}

Bridges are very important infrastructure facilities in a country. Among the various bridge types, steel bridges are popular due to their relatively light weight, ease of construction, and short building period [1]. In Taiwan, an island full of mountains and rivers, the government needs not only to build new bridges, but also to take care of more than 14,000 existing bridges that are in need of maintenance and repair [2].The intensive strikes of natural forces like typhoons and earthquakes in the recent years specially urged the Taiwanese government to conduct a comprehensive bridge health monitoring and assessment to ensure the safety of transportation.

One of the traditional methods to assess the quality of the coating on steel bridges surface is calculating the ratio of rust to the total area of coated surface. The American Society for Testing and Materials (ASTM) classified coating defect into 10 degrees according to the percentage of defected coating in the total coating area; for each degree of coating defects, it provides suggestions on how to rectify the defect [3].

However, it is difficult to determine rust zone and quantify the ratio by human vision. Digital image processing, therefore, provides a method to assess the rust defects and to identify objective and agreeable results for a maintenance organization to rely on. In North America, image processing for steel bridge coating inspection has been used since late 1990s [4-7].

Most of the previously developed systems segment images into two groups, rust and background, before assess the rust ratio. However, they are usually lack of the ability to recognize the intensities of rust defects. In order to provide a precise assessment, Yang et al. [7, 8] developed the adaptive ellipse approach (AEA) to classify the images into 
three parts: background colors, rust colors, and non-defined colors (such as mild rust colors, etc.). In AEA, background colors and rust colors are defined by the fundamental ellipse, and non-defined colors could be identified by adjusting fundamental ellipse. It is still insufficient in describing gradually changed colors and thus unable to classify those colors automatically. In this regard, this paper is focused on the development of a new approach that could classify the rust images into suitable number of groups for describing gradually changed colors automatically, which has been a hurdle in the two- or threegroup systems. To start, the image is segmented into several clusters based on the similarity of colors by KMeans algorithm, and the root-mean-square standard deviation (RMSSTD) is used to measure the similarity of each cluster. Artificial Neural Network is then adopted to develop the proposed method, a rust intensity recognition approach for steel bridge surface coating images based on Artificial Neural Networks (RIRAN). The segmentation results of RIRAN will be compared with the clustering results by statistical analysis.

\section{REGIONAL CLUSTERING}

The objective of image segmentation is to partition an image into multiple groups so that objects could be identified and the image could be easily analyzed [9]. Clustering analysis is one of the image segmentation techniques to groups multivariate objects into several partitions based on the similarity measure, such as distance between colors. Although clustering analysis is applied in image segmentation, its procedures often faced one unavoidable problems [10], defining the number of cluster.

Most of the previously developed steel bridge surface assessments grouped images into two clusters, rust and background. Yang et al. latterly classified the images into three parts: background colors, rust colors, and non-defined colors. However, these methods are lack of the ability to recognize rust intensity automatically, as the number of image clusters has been fixed.

In order to provide a flexible assessment, Chen [10] used the root-mean-square standard deviation (RMSSTD) [11, 12], defined in equation (1), to measure the standard deviation of all the variables. The smaller value of RMSSTD means more similarity within the groups.

$$
x=\sqrt{\frac{\sum_{j=1}^{k} S_{j}^{2}}{k}}
$$

where $k$ denotes the number of clusters and $S_{j}^{2}$ means the sample variance of cluster $j$.

Fig. $1 b$ shows the plot of RMSSTD value under different number of clusters using Fig. 1a as a model. The value of RMSSTD decreases along with the increased number of clusters.

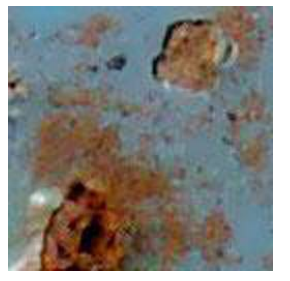

(a)

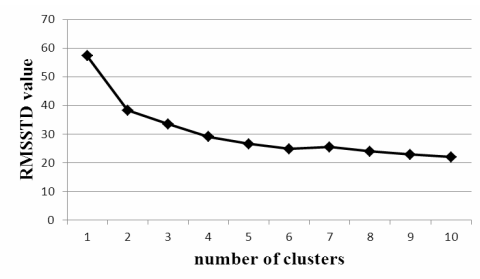

(b)
Fig. 1 The RMSSTD value varies under different number of clusters. (a) rust image; (b) the plot of the RMSSTD value.

In order to avoid too many trivial groups, Chen chose the largest difference of the RMSSTD value between $n$ and $n-1$ clusters as an appropriate number of clusters, $n$.

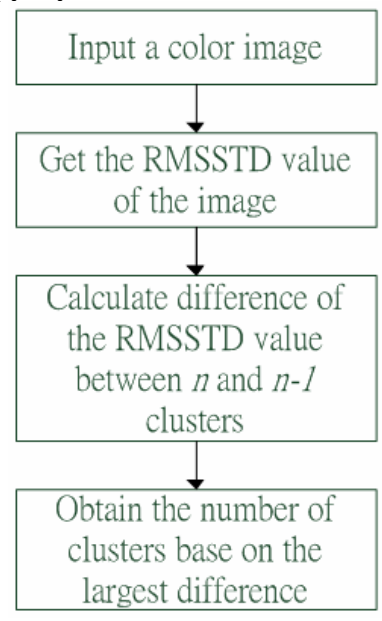

Fig. 2 Process of obtaining the appropriate number of clusters based on statistical analysis.

Fig. 2 shows the process of obtaining the number of clusters based on statistical analysis. According to Fig. 1b, the rust image is segmented into two clusters because the largest difference of the RMSSTD value is between 2 and 1 clusters. 
Fig. 3 shows the clustering result of Fig. 1a when the number of clusters is between 2 and 6 . According to a questionnaire survey, 4-cluster is commonly judged by observers. This experiment revealed the limitation of statistical analysis-based selection of best number of clusters, which always results in 2-cluster because the most significant difference is between the rust and background.

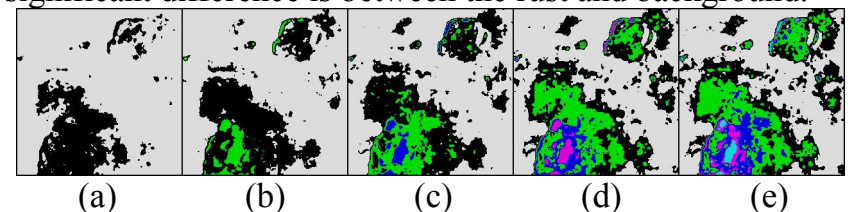

(a)

(b)

(c)

(d)

(e)

Fig. 3 (a)-(e) the clustering result under number of clusters 2-6.

\section{RUST INTENSITY RECOGNITION APPROACH BASED ON ARTIFICIAL NEURAL NETWORKS}

Because different observers may have different results of image clustering, we applied Artificial Neural Networks (ANNs) to analyze the RMSSTD value instead of statistical analysis. ANNs is an intelligent computational process which simulates the human behavior. Through the process of training, ANNs can accumulate previous experiences of making human-brain-like decisions and judgments [13]. ANNs has better capability to solve nonlinear problems and the problems which are hard to be defined by mathematical equations [7]. In this section, the training of the ANNs and the framework of RIRAN will be presented.

\subsection{Training of ANNs}

The ANNs plays an important role in the rust intensity recognition approach. It automatically generates suitable number of clusters to segment rust images. Fig. 4 shows the training process of ANNs.

To start, all pixels of a color image will be grouped into 210 clusters by K-Means algorithm based on the similarity of pixels under RGB space. We then used equation (1) to calculate the RMSSTD values of each number of clusters. The series of RMSSTD value was the input to the ANNs. Afterward, the questionnaire was set as the target value of the best clustering number of images. By comparing with the target value, the output from ANNs was adjusted.

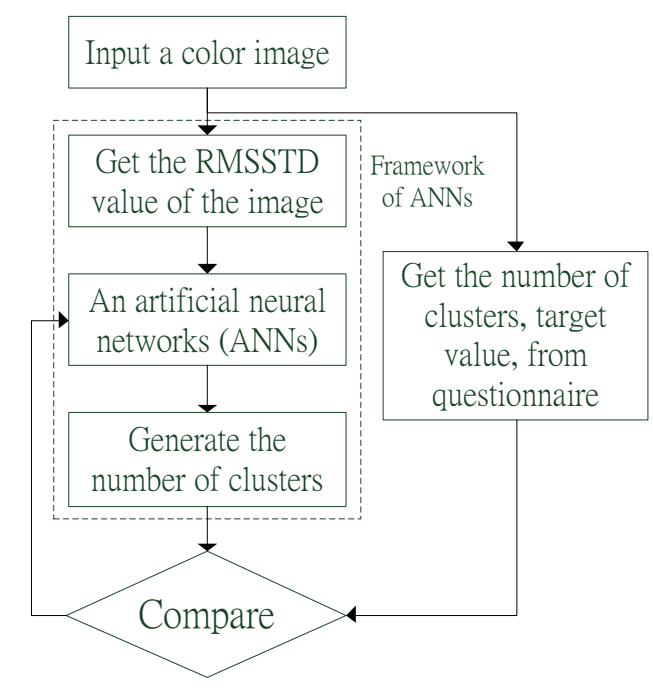

Fig. 4 Training process of ANNs.

The number of clusters of image is crucial for the segment approach to describe rust intensity. It is important to define a target value to train ANNs. However, the degree of gradually changed colors is a subjective description; different observers may have different color sensitivity and make divert results of clustering. In order to obtain the target value of the ANNs, one questionnaire was designed to collect the results of clustering numbers of images from a randomly chosen population. The questionnaire was consisted with 47 images with size of 256 x 256 pixels. Respondents judged the suitable number of clusters of image after a short glance not more than $10 \mathrm{sec}$. The mode value, which occurs most frequently in a data set in statistics, of each rust image was analyzed from the results of 41 respondents. The mode values were set as the target values of ANNs. The examples of questionnaire results and their clustering results by K-Means were shown in Fig. 5.

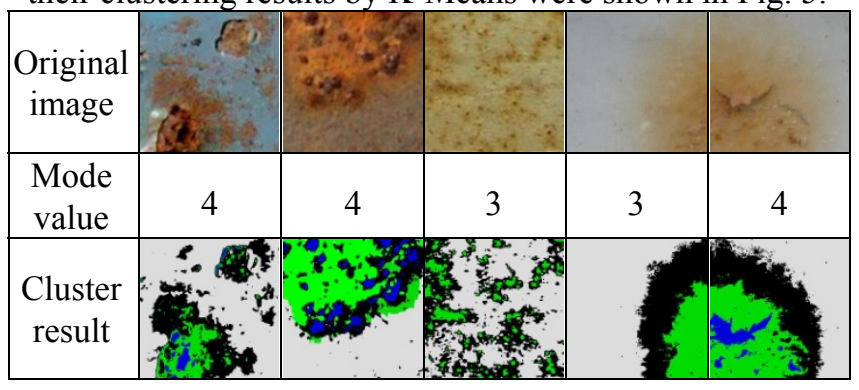

Fig. 5 The results of questionnaire.

3.2 Framework of RIRAN

Fig. 6 illustrates the framework and processing flow of the rust intensity recognition approach based on ANNs. 


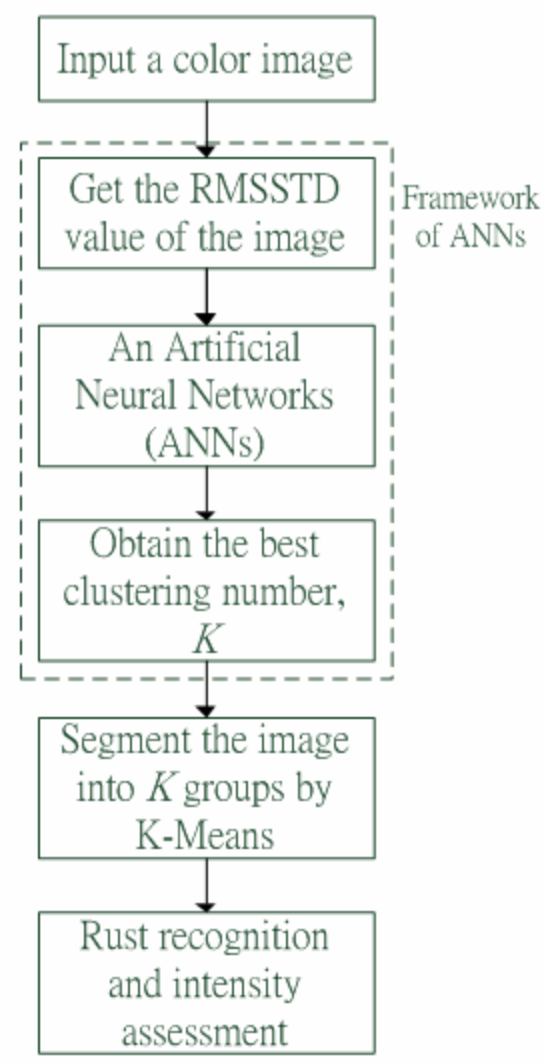

Fig. 6 Framework of RIRAN.

First, rust images were converted into RGB space. All pixels of the images were segmented into 2-10 clusters using K-Means algorithm based on the similarity of color feature. Second, we calculated the RMSSTD values of each number of clusters. The series of RMSSTD value were the input to the pretrained ANNs. The output, $K$, of ANNs would be the best clustering number. Finally, we applied K-Means algorithm to segment image into $K$ groups, and identified the rust groups by image segmentation methods base on color feature [14].

The main chemical compounds consisting rust are either $\mathrm{FeOOH}$ or $\mathrm{Fe} 2 \mathrm{O} 3 \cdot 3 \mathrm{H} 2 \mathrm{O}$ [15]. It is possible to define the rust in a specific color range by its color feature because the color difference between or within the two compounds is not significant. In order to define an appropriate color range for rust, we collected a set of sample color of rust. In this research, we collected several bridge images and cut off rust area by naked eyes. Pixels of these rust areas were collected in rust database. The color spectrum of the rust database is shown in Fig. 7. These data have been collected as a specified color range to define the color range of rust.

\section{Fig. 7 Color spectrum of rust database.}

We then evaluated the 'average' color of the set. The average would be the center of the specific color range. By determining whether the 'average' color of a group is within the specific color range or not, we could classify each group in an image. The group was classified as rust if it's 'average' color was inside the specified color range. Afterward, the rust percentage can be calculated after identifying rust groups.

\section{EXPERIENT RESULT}

This section shows the power of RIRN, and compares it with the results of clustering based on statistical analysis.

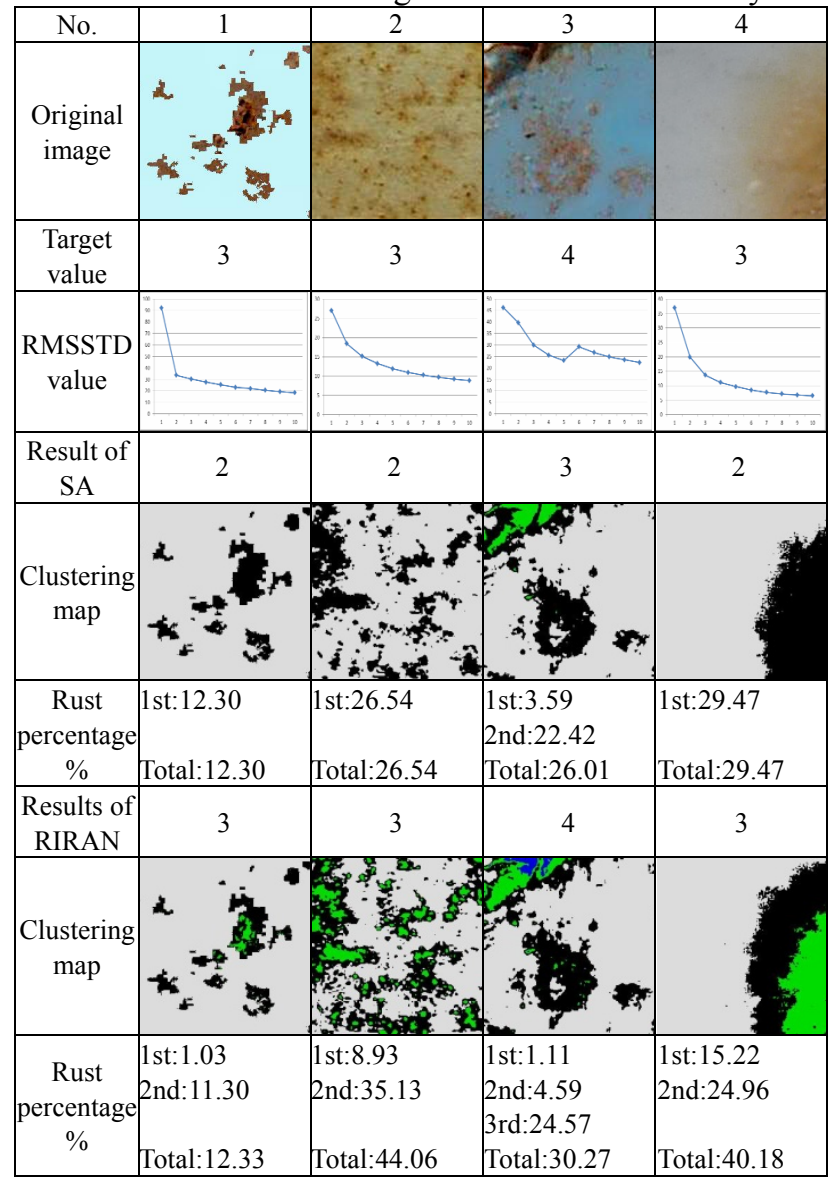

Fig. 8 recognition results by RIRAN and statistical analysis.

Fig. 8 indicates 4 recognition results from RIRAN or statistical analysis, respectively. The field 'Target value' presents the best clustering number derived from 
questionnaire survey, which is assumed to be accurate. The field 'Result of SA' shows the results by statistical analysis, and the field 'Results of RIRAN' shows the results of clustering by RIRAN.

According to Fig. 8, the images were mostly segmented into 2 clusters by statistical analysis because the most significant difference is between the rust and background. No. 3 was segmented into 3 clusters by statistical analysis because the color difference between heavy and light rust was obvious.

It is clear that RIRAN segmented all the images correctly according to Fig. 8. It is conceivable as the RIRAN method simulate human-brain-like decisions and judgments. Moreover, RIRAN can obtain more detailed information, such as percentage of each degree rust regions. The rust percentage of the image can be calculated by summing each degree rust regions. The rust percentages of No. 4 , for example, are $40.18 \%$ and $29.47 \%$ by RIRAN and statistical analysis, respectively. The lightly rusted area near the boundary of the rust can be identified by RIRAN, which statistical analysis cannot recognize RIRAN.

The capability of rust intensity recognition of RIRAN is better than that of statistical analysis. RIRAN has power for classify the rust images into suitable number of clusters for describing gradually changed colors automatically.

\section{CONCLUSIONS AND DISCUSSION}

The present report applied RMSSTD and ANNs to establish a rust intensity recognition approach, called RIRAN, for steel bridge surface coating quality assessment. RIRAN takes advantage of the different similarity of RGB color within the groups, and then judges the best number of clusters by ANNs.

RIRAN is able to classify the rust images into suitable number of clusters, which is closed to human decisions, for describing gradually changed colors. It overcomes the problem of defining clustering number prior to assessment in most of previously developed methods. Therefore, RIRAN is a more advanced method on steel bridge rust intensity recognition.
Although RIRAN is capable to describe gradually changed colors automatically, the rust segmentation is still based on K-Means algorithm, which is powerless to deal with images under environmental conditions such as nonuniform illuminations, low-contrast digital images, and noise on painting surface (Lee 2005). Instead of K-Means algorithm, it will be interesting to apply Box-and-EllipseBased ANFIS approach [16] or Support-Vector-MachineBased Method [17] to recognize the defects and assess the rust ratio. Both methods have better performance than KMeans in non-uniform illumination conditions. Despite this limitation, RIRAN is proven to be an effective and flexible method for steel bridge coating rust intensity assessment.

\section{REFERENCES}

[1] Wang, K.H., "Application of Artificial Neural Network to the Inspection of Steel Bridges Coating", in Department of Civil Engineering College of Engineering, National Chiao Tung University. p.12, 2002.

[2] M.O.T.C. statics of MINISTRY OF TRANSPORTATION AND COMMUNICATIONS. 2010/5/17; Available from: http://www.motc.gov.tw/mocwebGIP/wSite/lp?ctNode=17 $1 \&$ CtUnit=103\&BaseDSD=16\&mp=1, 2010.

[3] AbdelRazig, Y.A. and L.-M. Chang, "Intelligent Model for Constructed Facilities Surface Assessment”. Journal of Construction Engineering and Management, 126(6): p. 422-432, 2000.

[4] AbdelRazig, Y.A., "Construction quality assessment: A hybrid decision support model using image processing and neural learning for intelligent defects recognition". Purdue University: United States - Indiana, 1999.

[5] Chen, P.H. and L.M. Chang, "Intelligent steel bridge coating assessment using neuro-fuzzy recognition approach". Computer-Aided Civil and Infrastructure Engineering, 17(5): p. 307-319, 2002.

[6] Lee, S., L.M. Chang, and M. Skibniewski, “Automated recognition of surface defects using digital color image 
processing". Automation in Construction, 15(4): p. 540549, 2006.

[7] Yang , Y.C., "Smart Color Image Recognition for Steel Bridge Rust Inspection", in Department of Civil Engineering College of Engineering. National Taiwan University. p. 172, 2009.

[8] Chen, P.-H., Y.-C. Yang, and L.-M. Chang, "Automated bridge coating defect recognition using adaptive ellipse approach". Automation in Construction, 18(5): p. 632-643, 2009.

[9] Shapiro, L.G. and G.C. Stockman, "Computer vision”, Prentice Hall, 2001.

[10] Chen, L.-Y., "Color Image Segmentation Based on Markov Random Field", in Department of Statistics. National Cheng Kung University. p. 10-14, 2003.

[11] Jain, A.K., M.N. Murty, and P.J. Flynn, "Data clustering: a review.” ACM Comput. Surv., 31(3): p. 264323, 1999.

[12] Halkidi, M., Y. Batistakis, and M. Vazirgiannis, "Clustering validity checking methods: part II". SIGMOD Rec., 31(3): p. 19-27, 2002.

[13] Chen, P.-H., "Digital image recognition methods for infrastructure surface coating assessment". Purdue University: United States - Indiana, 2001.

[14] Gonzalez, R.C. and R.E. Woods, "Digital image processing". Upper Saddle River, NJ: Pearson/Prentice Hall, 2008.

[15] Dai, J.X., “Application of wavelet transform based intelligent image processing system to the inspection of steel bridges rusty areas", in Department of Civil Engineering College of Engineering. National Chiao Tung University. p. 89, 2004.

[16] Chen, P.-H., Y.-C. Yang, and L.-M. Chang, "Boxand-Ellipse-Based ANFIS for Bridge Coating Assessment". Journal of Computing in Civil Engineering, 24(5): p. 389398, 2010.

[17] Shen, H.K., P.H. Chen, and L.M. Chang. "SupportVector-Machine-Based Method for Automated Steel Bridge Rust Assessment". in The 5th Civil Engineering Conference in the Asian Region and Australasian Structural Engineering Conference. Sydney, Australia, 2010. 